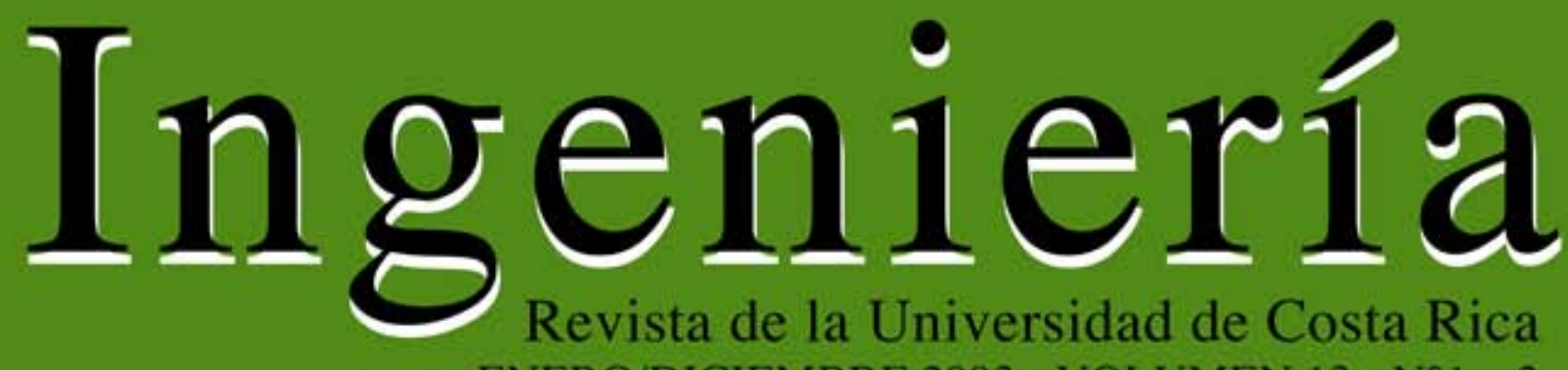
ENERO/DICIEMBRE 2003 - VOLUMEN 13 - N¹ y 2

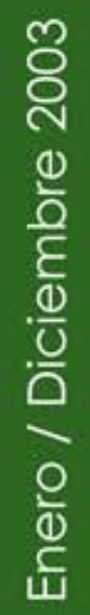

$\frac{m}{5}$
$\frac{c}{d}$
$\frac{5}{5}$
$\frac{\partial}{\circ}$

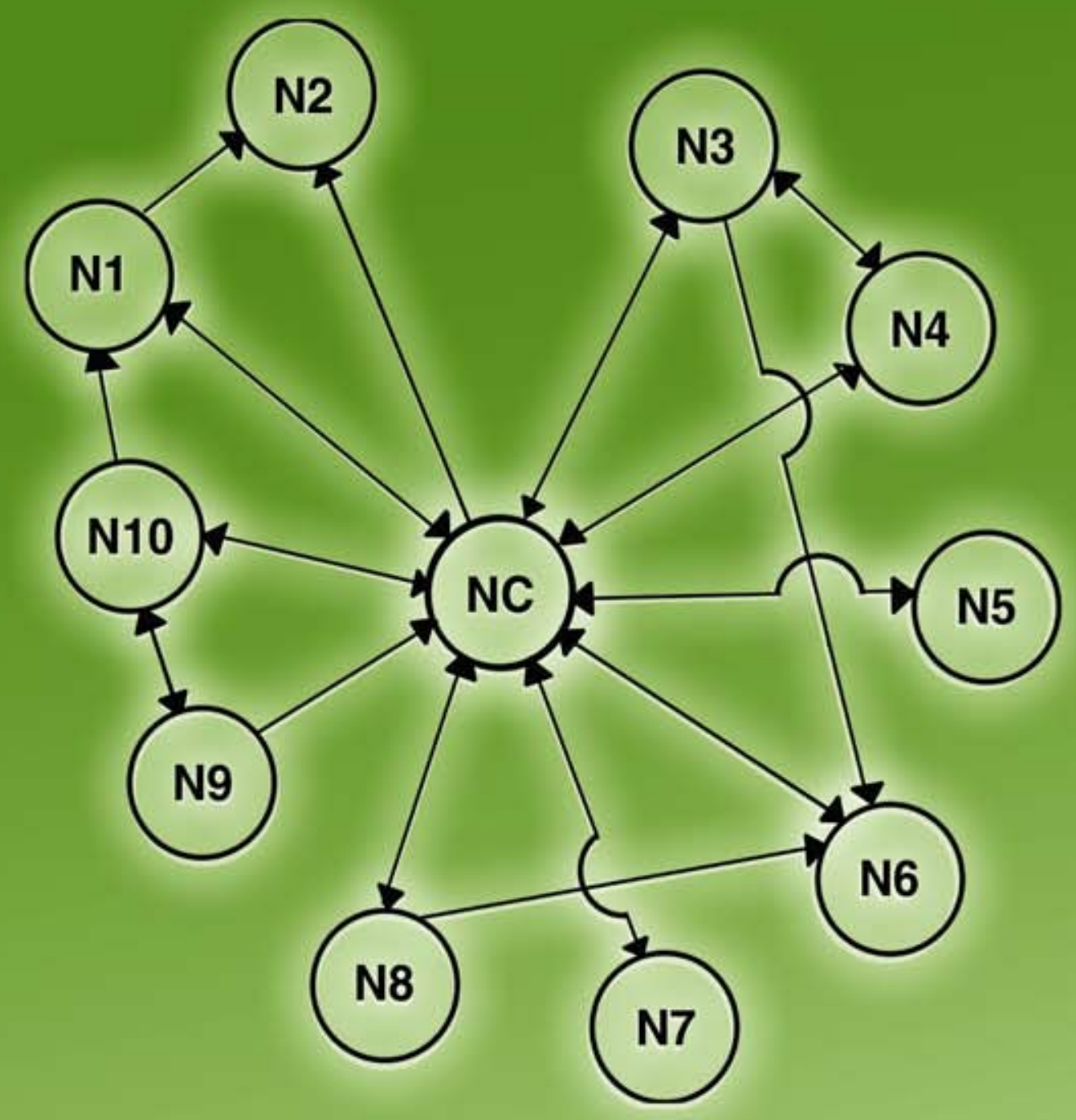

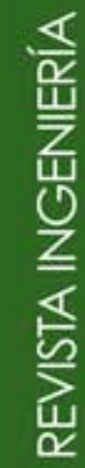




\title{
PROTECCIÓN PASIVA CONTRA INCENDIOS
}

\author{
Jhymer Rojas Vásquez
}

\begin{abstract}
Resumen
La protección de personas y bienes que no estén directamente expuestos al fuego puede conseguirse confinando el calor y el humo en su zona de origen hasta que sea controlado, se autoextinga o retrasándolo hasta que los ocupantes puedan trasladarse a un lugar seguro.

Las barreras y muros para estos efectos deben ser continuos y estables, capaces de resistir las fuerzas térmicas y físicas del fuego, así como las deformaciones y derrumbamientos que sufren las estructuras en la zona del incendio.

Estas notas pretenden dar una introducción a la protección pasiva contra incendios.
\end{abstract}

Palabras clave: propagación, retardantes, barreras, cortafuegos, sellantes, intumescencia, ASTM-E814

\begin{abstract}
Protection of people and goods that are not directly in contact with fire can be obtained by confining heat and smoke to the zone of origin until it has been controlled or extinguishes itself, or by delaying it until the occupants have moved to a safer location.

To accomplish this, the walls and barriers must be uninterrupted and stable, capable of resisting the thermal and physical forces due to fire, and also any structural failure in the fire zone.
\end{abstract}

These notes are an introduction to the topic of passive fire protection

Keywords: spread, retardant, barriers, firestops, sealant, intumescent, ASTM-E814

\section{INTENSIDAD DEL INCENDIO}

El desarrollo normal de un incendio puede caracterizarse por el avance de un frente de llamas sobre una superficie, por llamas que se generan en una fuente localizada, o ambas cosas a la vez. Aunque estos incendios no actúan de forma masiva sobre los muros cortafuegos, pueden propagarse a través de fallas o aberturas en ellos y disminuir su resistencia al fuego.

La intensidad máxima de transferencia térmica hacia las barreras cortafuegos surge cuando la ventilación alcanza precisamente el nivel suficiente para que la superficie del combustible controle la combustión. Los niveles más elevados de ventilación disipan más calor del incendio, debido al exceso de aire. Los niveles más bajos de ventilación desprenden menos calor de combustión y_descargan fuera de la zona del incendio más partículas de combustible y productos de pirólisis sin combustionar.

La posibilidad de rotura o destrucción de los muros cortafuegos puede surgir aunque haya transcurrido cierto tiempo desde el momento 
en que el incendio plenamente desarrollado comenzara a disminuir. Sin embargo, en muchos incendios reales, la actuación de los medios de extinción reduce esta amenaza.

La resistencia de un material al fuego se puede clasificar en función de tres criterios.

1. Estabilidad al fuego: resistencia mecánica de un material al estar en contacto o no con el fuego.

2. Parallamas: para ser clasificado como parallamas, el material tiene que ser estable al fuego, no permitir el paso a las llamas ni a los gases tóxicos.

3. Cortafuego: para ejercer esta función, el material necesita cumplir los anteriores criterios y además retardar la transmisión del calor entre recintos.

Los incendios en locales mal ventilados, como por ejemplo sótanos, bodegas o habitaciones interiores cerradas, a menudo producen bastante calor durante un largo período de tiempo, el cual penetra a través de los muros cortafuegos. Estos incendios suelen comenzar por combustión con llama y, según consumen el aire contenido en el local, pasan a la situación de combustión en brasas con producción esporádica e intermitente de llamas.

\section{CURVA NORMALIZADA DE TIEMPO-TEMPERATURA}

Para calcular la resistencia de los muros resistentes al fuego, se colocan probetas en hornos de ensayos $\mathrm{y}$ se los expone a un fuego cuya intensidad obedece a una curva de temperaturas que varía con el tiempo, denominada curva normalizada de tiempo-temperatura. La norma NFPA 251, presenta una curva de tiempo-temperatura (ver la Figura 1). La American Society for Testing Materials, (ASTM) adoptó la curva normalizada de tiempo-temperatura en 1918 y constituye, desde entonces, el fundamento de casi todos los ensayos de resistencia al fuego. El Cuadro
1 muestra los puntos que determinan la curva normalizada de tiempo y temperatura.

Cuadro 1. Puntos que determinan la curva normalizada de tiempo-temperatura (Tomado ASTM E-814)

Tiempo

Temperatura

$\left({ }^{\circ} \mathrm{C}\right)$

$\left({ }^{\circ} \mathbf{F}\right)$

5 minutos

538

10 minutos

704

1300

30 minutos

1550

1 hora

843

927

1700

2 horas

1010

1850

4 horas

1093

2000

8 horas

1260

2300

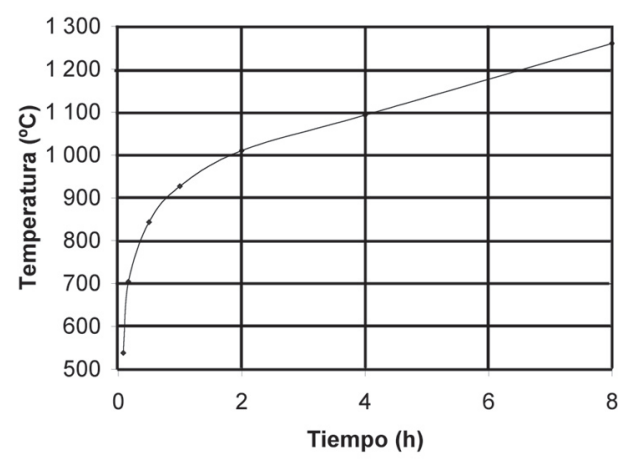

Figura 1. Curva normalizada tiempo temperatura

\section{PROPAGACIÓN DEL FUEGO}

Normalmente, el fuego se propaga en un edificio compartimentado a través de puertas abiertas, huecos de escaleras y cajas de ascensores sin cerrar, aberturas sin proteger en las barreras cortafuegos y espacios combustibles ocultos sin cortafuegos. Si la construcción y el mantenimiento de las barreras son adecuados y las aberturas tienen las protecciones necesarias, normalmente pueden contener los incendios. 
El fuego puede propagarse en sentido horizontal y vertical, más allá del recinto en que tuvo su origen a través de compartimentos o espacios que no contienen materiales combustibles. Los productos de pirólisis calientes sin quemar producidos por el fuego arden al mezclarse con aire limpio, mientras fluyen hacia el exterior y producen un movimiento amplio de las llamas. De este modo suelen propagarse los incendios a lo largo de los pasillos y en sentido vertical por huecos de escalera y pozos de ascensor abiertos. El acabado interior combustible en los pasillos y aberturas verticales, que por sí mismo no propagaría las llamas, al calentarse puede ocasionar productos de pirólisis. Estos productos agregados a los del incendio principal aumentan la intensidad y la longitud de las llamas. Al desarrollarse plenamente el fuego en el local, el incendio puede propagarse con gran rapidez a los espacios contiguos si no hay barreras cortafuegos. Este fue el caso del Gran Hotel MGM de Las Vegas. El fuego inició en el primer piso, sesenta y ocho personas murieron en el vigésimo tercer piso, cuando los humos y los gases letales viajaron libremente a través de juntas, ventilaciones, ductos y demás.

La capacidad de una barrera para detener el fuego puede considerarse un problema de probabilidad y fiabilidad semejante a los que presenta el funcionamientode equipos electrónicos, eléctricos y mecánicos. Mediante esta analogía, se puede considerar que los fallos de las barreras pueden clasificarse en tres categorías: fallos iniciales, fallos aleatorios y fallos por degradación. Las puertas que no estén cerradas o las aberturas sin protección originan los fallos iniciales. Los defectos de los materiales o de instalación, los fuegos de intensidad imprevista o los puntos débiles en el diseño sin identificar por los métodos normalizados de ensayos, causan los fallos aleatorios.

\section{ESPACIOS OCULTOS}

Los edificios pueden contener gran variedad de espacios ocultos detrás de tabiques, encima de techos suspendidos, en ductos para conducciones, debajo de tableros, debajo de pisos de salas de equipos de informática y en otros lugares.

Los incendios en los espacios ocultos arden sin que los ocupantes del edificio puedan detectarlos. La extinción manual de incendios en espacios ocultos puede tropezar con muchas dificultades, debido a los accesos limitados y a los problemas inherentes de ventilación. Los espacios ocultos verticales pueden actuar como chimeneas para propagar el incendio y los gases calientes.

El desarrollo del fuego y la propagación en los espacios ocultos combustibles representan una de las causas típicas de grandes pérdidas en incendios. El incendio puede tener su origen o propagarse hacia ellos y arder durante períodos prolongados, extendiéndose a lo largo de su recorrido.

El caudal del aire exterior, los materiales combustibles precalentados y los productos calientes de pirólisis producen, al combinarse, un crecimiento rápido del fuego, que puede salir del espacio oculto y propagarse ampliamente.

Los espacios huecos en falsos techos contienen con frecuencia aislamientos térmicos, cables de comunicación, tuberías de material plástico, etc. Si el aislamiento térmico es combustible, el fuego puede propagarse de manera semejante a como lo haría en espacios ocultos combustibles. No es probable que los materiales combustibles aislados propaguen el fuego horizontalmente; sin embargo, si están agrupados, pueden hacerlo.

Muchas centrales nucleares, tal y como lo recomienda la norma NFPA 850, utilizan actualmente cables eléctricos muy resistentes a la ignición. En los ensayos no propagaron el fuego horizontalmente, excepto en una configuración de bandejas múltiples apiladas. Asimismo, los ensayos indicaron que la utilización de revestimientos ignífugos puede inhibir eficazmente la propagación horizontal del fuego. 


\section{ABERTURAS VERTICALES}

Las aberturas verticales sin proteger han originado la pérdida de muchas vidas humanas en incendios. Esas aberturas pueden actuar como chimeneas; el humo, los gases calientes y los productos de pirólisis ardiendo fluyen en dirección ascendente por el efecto de tipo de chimenea, y se propagan horizontalmente al salir. Las aberturas, tales como pozos de ascensor, huecos de escalera, conductos de lavandería y pozos de ventilación, no pueden taparse en el nivel de los pisos, debido a sus funciones. Sin embargo, pueden protegerse con muros clasificados para resistir al fuego, impidiendo así la propagación del incendio; en el caso de vías de evacuación verticales, tienen que proporcionar un camino seguro para la salida de los ocupantes y para el acceso del personal del servicio de bomberos. Las vías de evacuación verticales interiores son, a menudo, la única salida al exterior, o a los pisos contiguos, de los ocupantes del edificio. La norma NFPA 101 estudia ampliamente las salidas en general y según las actividades realizadas en los edificios, tanto nuevos como ya construidos.

Las fosas utilizadas para cables eléctricos y de comunicaciones, canalizaciones o tuberías combustibles y demás materiales combustibles, necesitan normalmente protección mediante cerramientos y, a menudo, barreras cortafuegos en cada piso. La fiabilidad de la protección de las penetraciones de materiales combustibles a través de los muros del cerramiento será inferior normalmente a la de los materiales incombustibles. Si el fuego penetra en un ducto vertical que contenga materiales combustibles, puede propagarse rápidamente en dirección ascendente, produciendo mucho humo y extendiéndose, posiblemente, a otros pisos.

\section{COMPARTIMENTACIÓN EN RECINTOS}

Los objetivos de la compartimentación para confinar el incendio en una habitación o conjunto de habitaciones en que ha tenido su origen son, en general, los siguientes:
1. Aislar un espacio que tenga un nivel de riesgo de incendio más elevado que la zona circundante. Este sistema es aplicado generalmente alrededor de los cuartos o conjuntos utilizados como almacén de basuras y líquidos inflamables, hornos, laboratorios, talleres, cuartos de pintura, etc.

2. Reducir al mínimo el riesgo de pérdidas que puede sufrir el ocupante de un local debido a un incendio en otro espacio fuera de su área de control. Se consigue generalmente separando los apartamentos, conjuntos de oficinas, habitaciones, etc.

Además, la compartimentación ofrece una ventaja adicional porque limita el tamaño del incendio, reduce la cantidad de humo producido y facilita la extinción. Lo que da a los ocupantes del edificio, tiempo razonable para evacuar el inmueble con seguridad.

La compartimentación, debidamente proyectada e instalada, ha conseguido limitar muchos incendios a su lugar de origen. En varios incendios de edificios de gran altura, algunos ocupantes que no pudieron escapar a través de los pasillos fueron capaces de permanecer soportando grandes fuegos situados en apartamentos anexos a los suyos. La causa más frecuente de fracaso de este nivel de compartimentación ha sido una puerta sin picaporte o abierta. Las deficiencias de construcción son señaladas más comúnmente como causa de fracasos en edificios de construcción combustible.

Si una habitación está compartimentada, la ventilación probablemente no será suficiente para que el incendio prospere, a no ser que haya una ventana abierta o rota.

Asimismo, la compartimentación protege aquellos locales que contengan bienes valiosos u operaciones críticas de incendios en zonas circundantes. A menudo, los tabiques protegen las salas de equipos de informática, salas de mando, bóvedas, archivos, etc., de los incendios declarados en otras zonas del edificio. 


\section{PROTECCIÓN DE PASILLOS}

Los tabiques de pasillos resistentes al fuego suelen exigirse:

1. en la protección de los accesos a las salidas, de forma que se gane el tiempo suficiente para evacuar a los ocupantes, $\mathrm{o}$

2. para proporcionar a los bomberos un acceso protegido hasta el incendio. Además, al utilizarlos conjuntamente con separaciones cortafuegos entre habitaciones o grupos de habitaciones, las paredes del pasillo constituyen un elemento que impide la propagación del fuego.

Comúnmente se requiere que los tabiques de los pasillos posean una resistencia al fuego de una hora. En las aberturas que den a los pasillos hay que instalar, generalmente, puertas de autocerrado o de cierre automático, éstas por supuesto, deben ser resistentes al fuego. Dado que también hay que proteger a los pasillos del humo para permitir el acceso por ellos hasta la salida, se utilizan normalmente detectores de humo que accionen las puertas automáticas.

\section{NOTAS FINALES}

Aquí se ha mostrado brevemente en que consiste la protección pasiva contra incendios y su importancia. Este tema empieza a conocerse en Costa Rica principalmente por las nuevas construcciones, se espera que se tome conciencia y no se vea como un requisito más, ya que muchas vidas dependerán de ello. Por otro lado las compañías aseguradoras toman en cuenta la inversión en la seguridad contra incendios para establecer el monto de la póliza, por lo tanto no debe verse como un gasto adicional.

Se espera en próximos artículos tratar temas de sistemas de sellos contra fuego que se utilizan cuando se hacen penetraciones a través de los muros contra fuego. Asimismo se tratarán temas sobre los recubrimientos contra fuego, que constituye un campo muy amplio en el análisis de materiales en Ingeniería.

\section{BIBLIOGRAFÍA}

American Society for Testing Materials. (1983). ASTM E-84, Standard test method for surface burning characteristics of building materials. (Annual Book of ASTM Standards, Volume 04.07).

American Society for Testing Materials. (1983). ASTM E-119, Standard test methods for fire tests of building constrution and materials. (Annual Book of ASTM Standards, Volume 04.07).

American Society for Testing Materials. (1983). ASTM E-814, Standard test method for fire tests of through penetration firestops. (Annual Book of ASTM Standards, Volume 04.07).

Drysdale, D. (1998). An introduction to fire dynamics. (2a ed.). USA: John Wiley \& Sons Editorial.

National Fire Protection Association. (1999). NFPA 101, Code for safety to life from fire in buildings and structures. Spring Meeting Edition.

National Fire Protection Association. (1999). NFPA 220, Standard on types of building construction. Spring Meeting Edition.

National Fire Protection Association. (1999). NFPA 221, Standard for fire walls and fire barrier walls. Spring Meeting Edition.

National Fire Protection Association. (1999). NFPA 251, Standard methods of tests of fire endurance of building construction and materials. Spring Meeting Edition.

National Fire Protection Association. (1999). NFPA 255, Standard method of test of 
surface burning characteristics of building materials. Spring Meeting Edition.

National Fire Protection Association. (1999). NFPA 850, Recommended practice for fire protection for electric generating plants and high voltage direct current converter stations. Spring Meeting Edition.

National Fire Protection Association. (1999). NFPA 851, Recommended practice for fire protection for hydroelectric generating plants. Spring Meeting Edition.
National Fire Protection Association. (1999). NFPA 921, Guide for fire and explosion investigations. Spring Meeting Edition.

\section{SOBRE EL AUTOR}

\section{Jhymer Rojas Vásquez}

Profesor de la Escuela de Ingeniería Mecánica, Universidad de Costa Rica.

Máster en Ingeniería Mecánica, Univesidad de Costa Rica

Teléfonos:(506) 207-4548 Facsímil:207-5610

Correos eletrónicos: jhymer@fing.ucr.ac.cr jhymer@cfia.or.cr 